Olívia Maria de Paula Alves Bezerra 1

\title{
Talcose entre artesãos em pedra-sabão em uma localidade rural do Município de Ouro Preto, Minas Gerais, Brasil
}

\author{
Talc pneumoconiosis among soapstone \\ handicraft workers in a rural area of O uro Preto, \\ Minas Gerais, Brazil
}

Elizabeth Costa Dias 2

Márcio Antônio Moreira Galvão 1

Ana Paula Scalia Carneiro 3

\footnotetext{
1 Departamento de Nutrição Clínica e Social, Escola de Nutrição, Universidade Federal de Ouro Preto. Campus Morro do Cruzeiro, Ouro Preto, MG 35400-000, Brasil. ompab@uai.com.br magalvao@barroco.com.br 2 Departamento de Medicina Preventiva e Social, Faculdade de Medicina, Universidade Federal de Minas Gerais. Av. Alfredo Balena 190, 10 o andar, Belo Horizonte, MG 30130-100, Brasil. bethdias@medicina.ufmg.br 3 Ambulatório de Doenças Profissionais, Hospital das Clínicas, Universidade Federal de Minas Gerais. Alameda Álvaro Celso 55, 2o andar, Belo Horizonte, MG 30150-260, Brasil. apscalia@email.com
}

Abstract Talc pneumoconiosis in Brazil has received little research attention to date. The disease was investigated in Mata dos Palmitos, a district of Ouro Preto, Minas Gerais State, among soapstone handicraft workers. The district has some 180 inhabitants, of whom 108 are engaged in handicrafts production, while 15 alternate handicrafts production with work in the soapstone quarries. A clinical and occupational investigation was conducted, al ong with a respiratory symptoms standardized questionnaire, chest x-ray (ILO standard), and spirometry. Clinical data showed a low prevalence of respiratory complaints. Dyspnea was the most frequent symptom. Chronic bronchitis was identified in 12 adult workers. Chest x-rays showed evidence of small lung opacities in 5 workers, and in 11 there was suspicion of the same opacities. One worker showed a plaque-shaped pleural thickening. Seven workers showed abnormal spirometry. The soapstone dust composition showed breathable asbestos fibers from the amphibole group (tremolite-actinolite). The results suggest talc asbestosi s occurrence among soapstone handicraft workers.

Key words Pneumoconiosis; Talcosis; Occupational Health

Resumo A talcose é uma pneumoconiose ainda pouco estudada em nosso meio. Sua ocorrência foi investigada no Distrito de M ata dos Palmitos, Ouro Preto, Minas Gerais, Brasil, entre artesãos em pedra-sabão, que trabalham em produção de base familiar, de caráter informal. Na localidade, vivem cerca de 180 habitantes, dos quais 108 se ocupam com o artesanato em pedra-sabão e 15 alternam a produção de peças artesanais com as ativi dades nas minas. Foram realizados exames clínicos, enfatizando a história ocupacional; questionário padronizado de si ntomas respiratórios; radiografia de tórax (padrão OIT) e espirometria. Observou-se baixa prevalência de queixas respiratórias, e a dispnéia foi o sintoma mais freqüente. A ocorrência de bronquite crônica foi identificada em 12 trabal hadores adultos. O exame radiológi co de tórax evidenciou pequenas opaci dades pulmonares em cinco trabal hadores, e 11 foram consi derados suspei tos. U m trabal hador apresentou espessamento pleural em placa. Alterações na espirometria foram observadas em sete trabalhadores. O estudo da composição da poeira revelou a presença de fibras respi ráveis de asbesto do grupo dos anfi bóli os (tremolita-acti nolita). Esses resultados sugerem a ocorrência de talcoasbestose entre os artesãos em pedra-sabão.

Palavras-chave Pneumoconiose; Talcose; Saúde Ocupacional 


\section{Intro dução}

O esteatito, também conhecido como talco ou pedra-sabão, ocorre na região de Ouro Preto, Minas Gerais. É uma rocha metamórfica compacta, plástica, de baixa dureza e fina granulação, untuosa ao tato e facilmente riscada pela unha. Pode ser encontrada nas tonalidades de cinza, cinza-azulado, cinza-esverdeado e creme ou creme avermelhado, quando iniciado o processo de intemperização. Seu principal componente é o talco, um filossilicato de magnésio hidratado, podendo ocorrer também clorita, serpentina, magnesita, antigorita, enstatita e, ocasionalmente, quartzo, magnetita ou pirita.

O talco possui vários usos industriais, sendo empregado principalmente na indústria cerâmica, têxtil, farmacêutica, na produção de inseticidas, cosméticos, sabões, tintas, borrachas, papéis e refratários. A exposição ocupacional cumulativa à poeira de talco pode levar ao desenvolvimento da talcose, pneumoconiose decorrente da inal ação e conseqüente deposição de partículas respiráveis de talco nos alvéolos pulmonares. A doença, caracterizada pela fibrose pulmonar progressiva, irreversível, sem possibilidade de tratamento eficaz, pode manifestar-se vários anos após o início da exposição, mesmo depois de cessada a exposição (Jones et al., 1994); todavia, pode ser prevenida por meio de medidas eficazes de controle ambiental. No Brasil, é ainda pouco estudada, registrando-se apenas o relato da ocorrência de sete casos de talcose pulmonar por inalação ocupacional em moedores de talco (Chibante et al., 1990).

Em Ouro Preto, a utilização da rocha remonta ao século XVIII, quando passou a ser empregada na estatuária, na ornamentação das igrejas barrocas e na produção artesanal de objetos de cocção. Ainda hoje, a produção de objetos decorativos e utilitários em pedra-sabão constitui importante alternativa econômica para a população local excluída do mercado formal de trabalho. No subdistrito rural de Mata dos Palmitos, a população sobrevive, há mais de um século, da produção de artesanatos em pedra-sabão, sendo reconhecida a qualidade dos objetos lá criados. Essa produção, de caráter informal e de base familiar, ocorre em oficinas instaladas nas proximidades dos domicílios. A localidade possui 180 habitantes, dos quais 123 são artesãos. Indivíduos entre 7 e 17 anos representam aproximadamente $25 \%$ da força de trabal ho ocupada na produção de artesanato.

O processo de trabalho é rudimentar, com baixo grau de mecanização, observando-se a utilização de tornos e de serra elétricos em al- gumas oficinas, predominando o trabalho manual. $\mathrm{O}$ acesso dos artesãos à rocha vem sendo gradativamente dificultado ao longo dos anos; na atualidade, a matéria prima é comprada de empresas mineradoras que exploram as ocorrências na região. Alguns trabalhadores, ocasionalmente, alternam a produção de artesanatos com as atividades temporárias nas minas de talco, na fragmentação manual dos blocos da rocha extraídos mecanicamente.

No processo de produção das peças de artesanato ocorre intensa emissão de poeira mineral na atmosfera, à qual os trabal hadores e a população no entorno ficam expostos, especialmente crianças e bebês levados pelas mães para os locais de trabalho.

Estudo da matéria-prima e da poeira mineral gerada nas unidades de produção de artesanatos, desenvolvido por Bezerra (2002), incluiu as descrições petrográficas macroscópicas e microscópicas de amostras de rochas metaultramáficas coletadas em minas da região e nas unidades de produção; a determinação da concentração de poeira mineral (total, respirável e bulk, esta última visando à obtenção de massa significativa de poeira em suspensão na zona respiratória) e a análise química da poeira coletada para caracterização da sílica livre cristalina. Foi realizada a caracterização da exposição ocupacional por meio do monitoramento ambiental de fibras minerais respiráveis, com base em coleta exploratória e análise quantitativa e qualitativa de tais fibras.

As descrições petrográficas evidenciaram três tipos de rochas: talco xisto (duas amostras); anfibólio-clorita-talco xisto (três amostras) e anfibólio-clorita-talcito (seis amostras). Esses resultados indicam que os artesãos trabalham com pedra-sabão contaminada com formas asbestiformes do tipo anfibólio (tremolita-actinolita).

A caracterização da exposição ocupacional dos artesãos em relação à poeira mineral (total e respirável) não evidenciou a presença de massa de sílica livre cristalina $\left(\mathrm{SiO}_{2}\right)$ nas amostras coletadas. Também não foi obtida massa de $\mathrm{SiO}_{2}$ nas amostras bulk. A determinação quantitativa do talco e outros elementos na poeira não foi feita, uma vez que não são disponíveis metodologias analíticas laboratoriais validadas no Brasil. No entanto, imagens de microscopia eletrônica de varredura (MEV) de poeira respirável e total confirmaram que a poeira coletada era constituída basicamente de partículas de talco na forma de escamas, evidenciando, entre as mesmas, a presença de fibras de asbesto. Quanto à análise de fibras minerais respiráveis, o estudo concluiu que, ao se adotar 
critério técnico (AGCIH, 1999), o limite de tolerância para fibras respiráveis de asbesto foi ultrapassado em todos os pontos amostrados, evidenciando situação de risco grave iminente para a saúde dos artesãos, exigindo medidas de controle e intervenção imediata.

Concluindo, os resultados do estudo da rocha e da poeira de pedra-sabão gerada na produção de artesanato revelam que elas são compostas basicamente por talco, apresentando a poeira contaminação por fibras respiráveis de asbesto do grupo dos anfibólios (tremolita-actinolita). A exposição cumulativa a esta poeira pode causar a talcoasbestose, pneumoconiose decorrente da inalação de poeira de talco contaminado por asbesto (Feijin, 1986).

O presente estudo teve por objetivo conhecer a morbidade por talcose entre os artesãos de Mata dos Palmitos, como expressão de adoecimento relacionado ao trabalho, que obriga a adoção de modificações no processo produtivo capazes de eliminar/minimizar a emissão de poeira na atmosfera.

\section{Materiais e métodos}

Foi desenvolvido estudo epidemiológico descritivo, de delineamento transversal. A morbidade por talcose foi estudada adotando-se o procedimento usual para investigação de pneumoconioses, com base na comprovação da existência da poeira no local de trabal ho; na história ocupacional compatível com exposição à poeira; nos achados radiológicos no exame de tórax; na sintomatologia clínica e, se necessário, na realização de exames complementares (Algranti et al., 2003). Foram realizadas anamneses clínica e ocupacional, investigação dirigida de sintomas respiratórios, radiografia de tórax e avaliação da função pulmonar. Como os artesãos se recusaram a interromper a produção por períodos mais prolongados, todos os exames foram feitos na própria localidade, em salas de aula da escola da região, que foram adaptadas.

Tomou-se como base o universo de 123 artesãos que concordaram em participar do estudo, submetendo-se aos exames. O número de participantes variou em cada exame, sendo realizada anamnese ocupacional e aplicado o questionário padronizado de sintomas respiratórios nos 123 artesãos residentes na localidade. Em 89 deles (72,3\%), foi realizada anamnese clínica; em 117 (95\%), foi feito raio X de tórax, segundo a técnica padronizada pela Organização Internacional do Trabalho (OIT), e 27 (21,9\%) realizaram a avaliação da função pulmonar pela espirometria.
Para efeitos do estudo, os artesãos foram classificados por faixa etária segundo o Estatuto da Criança e do Adolescente (Brasil, 1990), que considera crianças indivíduos de até 12 anos incompletos e adolescentes, entre 12 anos completos e 18 anos incompletos.

$\mathrm{Na}$ anamnese clínica foram analisadas queixas espontâneas de problemas respiratórios freqüentes ou com características de cronicidade, manifestados como queixa principal. No exame físico foram ressaltados sinais auscultatórios pulmonares e expansibilidade torácica alterados.

Para a investigação dirigida da sintomatologia respiratória foi aplicado o questionário padronizado pela American Thoracic Soci ety ATS-DLD-78-A (Ferris, 1978). Apesar de existir versão própria desse questionário para crianças com até 13 anos de idade (a versão ATSDLD-78-C), ele não foi aplicado em virtude da discrepância que parte significativa das questões nele contidas guarda em relação à realidade das crianças de Mata dos Palmitos. Assim, optou-se por aplicar a versão ATS-DLD-78-A também em indivíduos com 13 anos ou menos, por se tratar de crianças trabalhadoras, em exposição ocupacional à poeira da pedra-sabão. O questionário foi aplicado por um dos pesquisadores, previamente treinado e auxiliado por médico do trabalho (que também se encarregou da interpretação dos dados obtidos), após realização de pré-teste. Foram analisados os sintomas tosse, expectoração (secreção proveniente dos pulmões), dispnéia e sibilância fora dos resfriados, sendo considerados sintomáticos os que apresentaram pelo menos um desses quadros. Considerou-se como apresentando tosse ou expectoração o indivíduo que respondeu positivamente quando perguntado se apresentava tais sintomas habitualmente. A intensidade da dispnéia foi graduada com base no questionário ATS-DLD-78-A (Ferris, 1978) e valorizada a partir do grau 2, que corresponde a andar mais devagar do que outra pessoa da mesma idade em superfície plana. Para indivíduos com 13 anos ou menos, não foram inquiridos os itens referentes à dispnéia (os critérios de quantificação desta para crianças são inadequados) e à história pregressa de enfisema (problema incomum nessa faixa etária).

Foi também investigada a presença de tabagismo entre artesãos com dez ou mais anos de idade. Identificou-se o tipo de fumo, a quantidade consumida por dia e o tempo de uso em anos. Considerou-se fumante o indivíduo que consumiu pelo menos um cigarro manufaturado ou $1 \mathrm{~g}$ de tabaco (fumo de rolo, Sabiá ou Trevo) por dia, no período de, no mínimo, um ano, 
e que preservava esse hábito na data da aplicação do questionário ou que havia deixado de fazê-lo nos seis meses anteriores. Indivíduos que mantiveram no passado esse nível de consumo e que haviam deixado de fazê-lo há mais de seis meses anteriores à aplicação do questionário foram considerados ex-fumantes. Para estimar a intensidade do consumo, foi calculado o índice Anos-Maço (AM) para fumantes atuais e ex-fumantes, através do produto do número de cigarros consumidos por dia (em maços de vinte unidades) pelo tempo de tabagismo (em anos). Para usuários de cigarro de palha ou cachimbo, considerou-se um grama de tabaco equivalente a um cigarro manufaturado (Algranti, 1991).

O questionário padronizado também contém questões relativas à história ocupacional, as quais foram aplicadas para todos os artesãos e complementadas mediante entrevista semiestruturada, realizada logo após sua aplicação.

As radiografias de tórax foram realizadas e interpretadas de acordo com as Diretrizes para Classifi cação Internacional de Radiografias de Pneumoconioses da OIT (ILO, 1980). As leituras foram feitas por três profissionais independentes (pneumologista, médico do trabalho e radiologista), considerados capacitados e experientes em leituras de raios $X$ para diagnóstico de pneumoconioses, segundo o padrão OIT, sendo um deles qualificado como " $\mathrm{B}$ " reader pelo National Institute for Occupational Safety and Health (NIOSH).

Foi obtida a mediana dos registros dos três leitores para qualidade da radiografia, profusão de pequenas opacidades, presença de grandes opacidades, presença de espessamento pleural e presença de calcificações pleurais, bem como para suas respectivas subcategorias. Para julgamento de forma e tamanho das opacidades, utilizou-se o critério de atribuição de pontos recomendado pela Classificação Internacional de Radiologias de Pneumoconioses (ILO, 1980). A classificação da profusão de pequenas opacidades foi empregada nas $12 \mathrm{sub}$ categorias: $0 /-; 0 / 0 ; 0 / 1 ; 1 / 0 ; 1 / 1 ; 1 / 2 ; 2 / 1 ; 2 / 2$; 2/3; 3/2;3/3; 3/ +. No Brasil, considera-se como caso de pneumoconiose a profusão mediana de opacidades igual ou superior a $1 / 0$, identificada por dois leitores qualificados e experientes, desde que comprovada a exposição e estabelecida história clínica e ocupacional. Como suspeita, considera-se a profusão mediana de opacidades igual a 0/ 1 (BRASI L, 1998). A presença de outros achados radiográficos importantes foi registrada por meio de símbolos (ILO, 1980), sendo valorizados quando apontados por, pelo menos, dois dos três leitores.
A avaliação da função pulmonar foi realizada por meio da espirometria, feita segundo as recomendações do I Consenso Brasileiro de Espirometria (Sociedade Brasileira de Pneumologia e Tisiologia, 1996). Utilizou-se espirômetro do tipo pneumotacógrafo, marca PuritanBennett, modelo PB100 S. Foram analisados os testes que apresentaram pelo menos uma curva aceitável, os quais foram posteriormente comparados com valores de referência para indivíduos de ambos os sexos de até 12 anos (Polgar \& Promadhat, 1971), para indivíduos do sexo masculino entre 13 a 24 anos e do sexo feminino entre 13 e 19 anos (Knudson et al., 1976) e para indivíduos do sexo masculino de 25 a 78 anos e do sexo feminino de 20 a 76 anos (Pereira et al., 1992), de acordo com idade, altura e sexo de cada indivíduo examinado.

Todos os princípios éticos relacionados a estudos com seres humanos foram cumpridos, sendo o projeto de pesquisa aprovado pelo Comitê de Ética em Pesquisa com Seres Humanos da Universidade Federal de Ouro Preto.

\section{Resultados}

A Tabela 1 apresenta algumas características demográficas e da exposição à poeira da pedrasabão dos artesãos de Mata dos Palmitos.

Dos 89 artesãos submetidos à anamnese clínica, 74 (83,1\%) eram adultos e 15 (16,9\%), adolescentes. Oito adultos e um adolescente $(10,1 \%)$ referiram problemas respiratórios freqüentes como queixa principal. Ao exame físico, foram identificadas alterações na ausculta pulmonar em seis trabalhadores adultos $(6,7 \%)$, sendo crepitações bilaterais em um, sibilos em dois e murmúrio vesicular diminuído em três, além de expansibilidade torácica diminuída em dois artesãos (2,2\%).

Entre os 123 artesãos que responderam ao questionário padronizado de sintomas respiratórios, 31,7\% apresentaram pelo menos um dos sintomas analisados, sendo, assim, considerados sintomáticos. Os principais achados relativos à sintomatologia respiratória são apresentados na Tabela 2.

A combinação de tosse e expectoração (secreção proveniente dos pulmões) pela manhã, por período superior a três meses ao ano, presente por no mínimo dois anos consecutivos, configura o diagnóstico clínico de bronquite crônica (BC). Esse diagnóstico foi identificado em 12 artesãos adultos, representando 13\% dos 92 adultos que responderam ao questionário.

Identificou-se o hábito de consumir, além de cigarros manufaturados, cigarros de palha 
entre homens e mulheres adultas que responderam ao questionário. Entre mulheres idosas, foi observado o hábito de fumar cachimbo. Entre adolescentes e crianças com mais de dez anos de idade não foram identificados fumantes. A quantidade de fumantes e de ex-fumantes correspondeu, respectivamente, a 35,7\% e $16,5 \%$ dos inquiridos. O índice Anos- $M$ aço variou, respectivamente, entre 1 e 99 (mediana = 10,5 ) e entre 1 e 30 (mediana $=10,5$ ).

Foram radiografados 117 artesãos (87 adultos, 15 adolescentes e 15 crianças) com idade entre 7 e 82 anos (mediana $=25$ ). Cinco radiografias (4,3\%) apresentaram mediana da profusão de pequenas opacidades igual ou superior a $1 / 0$, correspondendo a casos de pneumoconiose, e 11 (9,4\%) apresentaram mediana da profusão de pequenas opacidades igual a $0 / 1$, correspondendo a suspeitas da doença. Não foram identificadas confluências de nódulos e grandes opacidades pulmonares. As principais alterações radiográficas pulmonares encontradas são apresentadas na Tabela 3.

Em três indivíduos adultos, nos quais não foram observadas pequenas opacidades pulmonares ( profusão $=0 / 0$ ), o seio costofrênico estava obliterado unilateralmente.
Tabela 1

Características demográficas e da exposição à poeira da pedra-sabão dos 123 artesãos de Mata dos Palmitos, O uro Preto, Minas Gerais, Brasil.

\begin{tabular}{lc}
\hline Características demográficas e da exposição & Dados encontrados \\
\hline Idade média (anos)1,2 & \\
adultos $(n=92)$ & $35,2 \pm 15,8$ \\
adolescentes $(n=16)$ & $9,2 \pm 1,5$ \\
crianças $(n=15)$ & \\
Sexo & $52 \%$ \\
masculino $(n=64)$ & $48 \%$ \\
feminino $(n=59)$ & \\
Tempo de exposição ocupacional (anos) $)^{3,4}$ & 10 \\
adultos $(n=92)$ & $4,9 \pm 2,7$ \\
adolescentes $(n=16)$ & $2,1 \pm 1,2$ \\
crianças $(n=15)$ &
\end{tabular}

1 Classificação segundo o Estatuto da Criança e Adolescente.

2 Média e desvio-padrão. A idade dos 123 artesãos variou entre 7 e 82 anos (mediana $=25$ ).

3 Média e desvio-padrão. Para adultos, o valor refere-se à mediana do tempo de exposição (que variou entre 1 e 60 anos). 0 tempo de exposição ocupacional dos 123 artesãos variou entre 1 e 60 anos (mediana $=10$ ).

4 Todos os artesãos negaram exposição ocupacional anterior a outros tipos de poeira.

Prevalência de sintomas respiratórios entre artesãos em pedra-sabão por faixa etária.

Mata dos Palmitos, O uro Preto, Minas Gerais, Brasil.

\begin{tabular}{|c|c|c|c|c|c|c|c|c|}
\hline \multirow[t]{2}{*}{$\begin{array}{l}\text { Sintomas } \\
\text { respiratórios }\end{array}$} & \multicolumn{4}{|c|}{$\begin{array}{l}\text { Classificação etária } \\
\text { Adolescentes }(n=16)\end{array}$} & Crianças & 15) & \multicolumn{2}{|c|}{ Total $(n=123)$} \\
\hline & Freqüência & $\%$ & Freqüência & $\%$ & Freqüência & $\%$ & Freqüência & $\%$ \\
\hline Assintomáticos & 55 & 59,8 & 14 & 87,5 & 15 & 100,0 & 84 & 68,3 \\
\hline Sintomáticos & 37 & 40,2 & 2 & 12,5 & - & - & 39 & 31,7 \\
\hline Tosse & 14 & 15,2 & 1 & 6,3 & - & - & 15 & 12,2 \\
\hline Expectoração & 22 & 23,9 & - & - & - & - & 22 & 17,9 \\
\hline Dispnéia & 35 & 38,0 & 1 & 7,71 & NSA & NSA & 36 & 34,32 \\
\hline \multicolumn{9}{|c|}{ Grau de dispnéia3 } \\
\hline 1 & 5 & 14,3 & - & - & NSA & NSA & 5 & 13,9 \\
\hline 2 & 10 & 28,6 & - & - & NSA & NSA & 10 & 27,8 \\
\hline 3 & 14 & 40,0 & 1 & 100,0 & NSA & NSA & 15 & 41,6 \\
\hline 4 & 1 & 2,9 & - & - & NSA & NSA & 1 & 2,8 \\
\hline 5 & 5 & 14,3 & - & - & NSA & NSA & 5 & 13,9 \\
\hline Sibilância & 74 & 7,6 & 1 & 6,3 & - & - & 8 & 6,5 \\
\hline
\end{tabular}

1 Percentual calculado considerando-se o número de indivíduos com idade superior a 13 anos $(\mathrm{n}=13$ ).

2 Percentual calculado considerando-se o número de indivíduos com idade superior a 13 anos $(n=105)$,

já que três adolescentes e 15 crianças com idade igual ou inferior a 13 anos não foram inquiridos

sobre ocorrência do sintoma dispnéia.

3 Percentual calculado considerando-se o número de dispnéicos em cada faixa etária.

4 Cinco artesãos relataram ter tido pelo menos uma crise de sibilância com dispnéia. 
Principais alterações radiog ráficas pulmonares entre artesãos, segundo a mediana das leituras.

Mata dos Palmitos, O uro Preto, Minas Gerais, Brasil.

\begin{tabular}{|c|c|c|}
\hline Alterações radiográficas pulmonares & Casos $(n=5)$ & Suspeitos $(n=11)$ \\
\hline \multicolumn{3}{|l|}{ Profusão de pequenas opacidades } \\
\hline $0 / 1$ & NSA & 11 \\
\hline $1 / 0$ & 4 & NSA \\
\hline $2 / 2$ & 1 & NSA \\
\hline \multicolumn{3}{|l|}{$\begin{array}{l}\text { Formas e tamanhos predominantes } \\
\text { das pequenas opacidades }\end{array}$} \\
\hline \multicolumn{3}{|l|}{ Regulares (arredondadas) } \\
\hline $\mathrm{p}$ & 1 & 4 \\
\hline q & - & 1 \\
\hline r & - & - \\
\hline \multicolumn{3}{|l|}{ Irregulares (lineares) } \\
\hline $\mathrm{s}$ & 3 & 4 \\
\hline $\mathrm{t}$ & 1 & - \\
\hline u & - & - \\
\hline Mistas1 & - & 2 \\
\hline \multicolumn{3}{|l|}{ Distribuição das pequenas opacidades² } \\
\hline Zonas superior, média e inferior & 3 & 6 \\
\hline Zonas média e inferior & 1 & 4 \\
\hline Zona inferior & 1 & 1 \\
\hline \multicolumn{3}{|l|}{ Pleura } \\
\hline Normal & 4 & 8 \\
\hline Alterada & - & 13 \\
\hline A investigar 4 & 1 & - \\
\hline Seios costofrênicos obliterados unilateralmente & - & 2 \\
\hline \multicolumn{3}{|l|}{ Outros achados (símbolos) 5} \\
\hline Alterações na forma e tamanho do coração (co) & 2 & 4 \\
\hline Enfisema (em) & - & 1 \\
\hline O utras alterações significativas (od) & 16 & 47 \\
\hline Espessamento pleural da cisura interlobar (pi) & 1 & - \\
\hline
\end{tabular}

1 Nos dois casos houve predominância de lesões irregulares sobre as regulares.

2 Pequenas opacidades distribuídas bilateralmente, tanto nos casos quanto nos suspeitos.

3 Espessamento pleural do tipo placa, largura B, extensão 1, com ausência de calcificações.

4 Imagem sugestiva de espessamento pleural difuso bilateral em um adulto, apontado por um dos leitores

e com sugestão de investigação complementar por outro.

5 Identificados somente em adultos.

6 Linfonodos hilares aumentad os e calcificados.

7 Granuloma no lobo superior esquerdo em três artesãos e espessamento de paredes brônquicas em um artesão.

Quanto à função pulmonar, foram realizadas 99 espirometrias no próprio local de estudo, das quais apenas 27 foram consideradas tecnicamente aceitáveis. A mediana da idade dos artesãos efetivamente examinados foi igual a 32 (entre 9 e 73 anos). Sete artesãos $(25,9 \%)$ apresentaram espirometrias alteradas, das quais três $(42,9 \%)$ com padrão obstrutivo e quatro $(57,1 \%)$ com padrão restritivo.

\section{Discussão}

Os achados da anamnese clínica, realizada em 89 artesãos, possivelmente não guardam relação com a exposição à poeira, especialmente a expansibilidade torácica e o murmúrio vesicular diminuídos, já que tais sinais normal mente se manifestam em estágios mais avançados de pneumoconiose, quando ocorre confluência de nódulos, o que não foi constatado no pre- 
sente estudo. Os problemas respiratórios freqüentes relatados podem estar associados a outros fatores ambientais, como, por exemplo, o tabagismo (Jones et al., 1994).

A prevalência de sintomatologia respiratória encontrada no presente estudo $(31,7 \%)$ pode ser considerada baixa, se comparada a estudo brasileiro sobre doenças respiratórias ocupacionais utilizando questionário padronizado de sintomas respiratórios (Pivetta \& Botelho, 1997). Normal mente a sintomatologia respiratória não se associa à exposição a poeiras minerais, mesmo entre indivíduos que apresentam algum grau de comprometimento pulmonar (Jones et al., 1994). Quando presente, geralmente está relacionada ao tabagismo, considerado principal fator de risco (Botelho et al., 1989), o que possivel mente explica a predominância de sintomas respiratórios entre trabaIhadores adultos neste estudo.

O sintoma mais freqüente foi dispnéia, encontrado em $34,2 \%$ dos inquiridos para este sintoma e em $92,3 \%$ dos sintomáticos. Entre estes últimos, 86,1\% apresentaram dispnéia de grau $\geq 2$, sendo esta magnitude importante, já que, além de constituir o sintoma mais prevalente entre trabalhadores em exposição ocupacional a poeiras minerais, embora nem sempre presente, especialmente em casos incipientes de pneumoconiose, costuma manifestar-se em indivíduos expostos a poeiras contendo fibras de asbesto, sobretudo entre aqueles com pneumoconiose estabelecida (Parkes, 1973). Expectoração ocorreu em 17,9\% dos artesãos inquiridos, seguida pela tosse, presente em 12,2\% deles. Alguns artesãos que relataram expectoração negaram a ocorrência simultânea de tosse freqüente, o que possivelmente refere-se a uma situação de produção de secreção nas vias aéreas superiores.

BC foi identificada em $13 \%$ dos adultos que responderam ao questionário. No Brasil, existem poucos estudos de base populacional nos quais a prevalência de BC foi avaliada. Estudo desenvolvido por Menezes et al. (1994) em Pelotas, Rio Grande do Sul, relatou uma prevalência de $12,7 \%$ entre indivíduos com mais de quarenta anos de idade. Ramos (1983), ao estudar a prevalência de sintomas respiratórios em uma amostra de 3.353 indivíduos maiores de três anos de idade na cidade de Ribeirão Preto (São Paulo), encontrou uma prevalência de BC da ordem de 5,5\% e 3,3\% respectivamente para homens e mulheres. A prevalência encontrada no presente estudo (13\%) aproxima-se da encontrada em outros envolvendo trabalhadores expostos a poeiras minerais (Pivetta \& Botelho, 1997). O tabagismo é considerado o fator mais importante na etiologia da BC. No entanto, a presença dessa doença como decorrência da exposição ocupacional a poeira de talco puro tem sido relatada (Isaza et al., 1988; Wegman et al., 1982).

A prevalência encontrada de sibilância fora dos resfriados (6,5\%), indicativo de suspeita de asma, pode ser considerada baixa se comparada, por exemplo, à encontrada por I saza et al . (1988) entre mineiros de talco colombianos, da ordem de $52 \%$.

A prevalência de tabagismo atual encontrada $(35,7 \%)$ coincide com dados da população brasileira, que oscilam entre $32 \%$ e $42 \%$ (Moreira \& Fuchs, 1995). Já a mediana do índice Anos-Maço encontrada para o grupo de fumantes atuais $(19,5)$ pode ser considerada elevada (Franco et al., 1989). Alguns autores têm sugerido que o hábito de fumar parece exacerbar os efeitos das partículas minerais nos pulmões. Wegman et al. (1982), por exemplo, sugeriram que a inalação de talco puro em não fumantes não chega a causar as anormalidades clínicas e radiológicas características da talcose.

Foi encontrada uma prevalência de 4,3\% de casos de pneumoconiose por exposição ocupacional ao talco no presente estudo. Apesar de a prevalência da doença no Brasil ser desconhecida, observa-se que esse índice aproxima-se do encontrado por Alhmark et al. (1958) entre 110 mineiros de talco suecos (da ordem de $4,5 \%$ ), cujo tempo médio de exposição era superior a vinte anos. Outros estudos relatam prevalências superiores à encontrada no presente estudo (Gamble et al., 1979; Isaza et al., 1988; Wegman et al., 1982). Porém, é preciso considerar que os estudos citados referem-se à extração ou processamento industrial do talco, atividades em que a exposição adquire características diferentes da verificada em Mata dos Palmitos.

Suspeita da doença foi identificada em $10,1 \%$ dos radiografados, incluindo dois adolescentes de 13 e 14 anos de idade, o que possivelmente também está relacionado, além da exposição ocupacional precoce, à exposição ambiental, conforme já citado.

Quanto à forma e distribuição das pequenas opacidades, tal como encontrado no presente estudo, tem sido relatada a presença de opacidades regulares $(p, q, r)$, irregulares $(s, t$, u), ou mistas em indivíduos expostos ao talco, sendo as lesões regulares encontradas principalmente nas zonas pulmonares superiores e médias, e as irregulares, na zona inferior, mesmo em indivíduos expostos à poeira de talco puro (Feijin, 1986; M organ \& Seaton, 1995). 
Foi identificado espessamento pleural em placa em um artesão (profusão $=0 / 1$ ) e indicada essa possibilidade em outro (profusão = 1/0). A formação de espessamento pleural em trabalhadores expostos a poeiras de talco contaminado por anfibólios tem sido relatada. Normalmente surge após longo período de latência, não se associando a alterações funcionais, a não ser quando muito extenso (Feijin, 1986; Jones et al., 1994). A obliteração de seios costofrênicos, encontrada em cinco artesãos adultos, dois deles considerados suspeitos da doença e três que não apresentaram pequenas opacidades pulmonares, adquire significado ao se considerar que esses trabalhadores estão expostos a fibras de asbesto. O espessamento pleural difuso geralmente se inicia nessa região, acometendo pleura visceral e parietal em direção ao ápice pulmonar (FUNDACENTRO, 1994). Como a contaminação da poeira da pedra-sabão por fibras respiráveis de anfibólios (tremolita-actinolita) foi identificada em Mata dos Palmitos, torna-se conveniente aprofundar a investigação de possíveis casos de lesão pleural em estudos posteriores, através de propedêutica complementar.

Segundo a literatura, outros achados radiográficos identificados (co, em, od e pi) poderiam, em tese, estar associados à exposição a poeiras minerais e à ocorrência de pneumoconiose, contudo não se pode descartar a contribuição de outros fatores, como, por exemplo, o tabagismo ou história pregressa de doença pulmonar de origem infecciosa. Novos estudos deverão ser realizados para o melhor conhecimento dessas questões.
Quanto às espirometrias, o número reduzido de exames constitui fator limitante para a interpretação dos resultados encontrados, especialmente quanto à sua possível associação com fatores de risco como exposição à poeira e tabagismo. Chama a atenção, no entanto, a predominância do padrão restritivo entre os casos considerados alterados, já que tal padrão entre trabalhadores expostos à poeira de talco tem sido descrito em fases mais avançadas da pneumoconiose (Jones et al., 1994).

\section{Conclusões}

Os resultados do estudo petrográfico e da caracterização da exposição ocupacional, a história ocupacional e os resultados da avaliação clínico-radiológica sugerem que as alterações radiológicas pulmonares verificadas entre os artesãos de M ata dos Palmitos podem ser consideradas como talcoasbestose. A identificação de um caso de espessamento pleural - sugestivo da exposição a fibras respiráveis de asbesto -, assim como de outro caso provável desse tipo de lesão reforça essa possibilidade. Entretanto, do ponto de vista da abordagem individual, a definição precisa do tipo de pneumoconiose somente será possível pela realização de biópsia pulmonar, considerada "padrão ouro" para o diagnóstico de pneumoconioses.

A gravidade do problema e suas repercussões sobre a saúde e a qualidade de vida dos artesãos em pedra-sabão, muitos deles, ainda crianças, exigem a adoção imediata de medidas de proteção e de atenção à saúde desses trabalhadores.

\section{Agradecimentos}

Os autores agradecem ao $\mathrm{Dr}$. René Mendes, ao $\mathrm{Dr}$. Eduardo Algranti e ao Dr. Sandro Santos Fenelon pelas leituras das radiografias, comentários, críticas e sugestões. 


\section{Referências}

ACGIH (American Conference of Governmental Industrial Hygienists), 1999. Limites de Exposição para Substâncias Químicas e Agentes Físicos e Índices Biológi cos de Exposição. São Paulo: Associação Brasileira de Higienistas Ocupacionais.

ALGRANTI, E., 1991. Doenças Respiratórias Associadas à Mineração do Carvão: Estudo de Coorte de Cinco Anos. Tese de Doutorado, São Paulo: Faculdade de Saúde Pública, Universidade de São Paulo.

ALGRANTI, E.; CAPITANI, E. M.; CARNEIRO, A. P. S. \& SALDIVA, P. H. N., 2003. Patologia respiratória relacionada com o trabalho. In: Patologia do Trabalho (R. Mendes, org.), pp. 1329-1397, Rio de Janeiro: Atheneu.

ALHMARK, A.; BRUCE, T. \& MYSTROIN, A., 1958. Pneumoconiosis (talcosis) in soapstone workers. Nordisk Medicin, 59:287-288.

BEZERRA, O. M. P. A., 2002. Condi ções de Vida, Produção e Saú de em uma Comuni dade de Mineiros e Artesãos em Pedra-Sabão em Ouro Preto, Minas Gerais: Uma Abordagem a partir da Ocorrência de Pneumoconioses. Tese de Doutorado, Belo Horizonte: Escola de Veterinária, Universidade Federal de Minas Gerais.

BOTELHO, C.; GUEDES-BARBOSA, L. S. \& JARDIM, J. R. B., 1989. Sintomas respiratórios, espirometria e tabagismo em adultos - Cáceres, MT. Jornal de Pneumologia, 15:74-78.

BRASIL, 1990. Lei no 8.069, de 13 de julho de 1990. Estatuto da Criança e do Adol escente. 15 Julho 2001 বttp:// www.lawnet.com.br/codigos_2.htm>.

BRASIL, 1998. Ordem de serviço no 609, de 5 de agosto de 1998. Aprova norma técnica sobre pneumoconioses. Brasília: Diário Oficial da União, 19 ago.

CHIBANTE, A. M. S.; PADILHA, C. P.; BETHLEN, E. P.; DIAS, R. M.; OLIVEIRA, C. A. S. \& MAGARÃO, S. L., 1990. Pneumoconiose dos moedores de talco: estudo de sete casos. Jornal de Pneumologia, 16:5761.

FEIJIN, D. S., 1986. Talc: Understending its manifestations in the chest. American Journal of Roentgenology, 146:295-301.

FERRIS, B. G., 1978. Epidemiology standardization project II. American Review of Respiratory Diseases, 118:7-53.

FRANCO, E. L.; KOWALSKI, L. P.; OLIVEIRA, B. V.; CURADO, M. P.; PEREIRA, R. N.; SILVA, M. E.; FAVA, A. S. \& TORLONI, H., 1989. Risk factors for oral cancer in Brazil: A case-control study. International Journal of Cancer, 43:992-1000.

FUNDACENTRO (Fundação Jorge Duprat Figueiredo de Segurança e Medicina do Trabalho), 1994. Leitura Radiológica de Pneumoconioses. 2a Ed., São Paulo: FUNDACENTRO.

GAM BLE, J. F.; FELLNER, W. \& DIMEO, M. J., 1979. An epidemiologic study of a group of talc workers. American Review of Respiratory Diseases, 119:741753.
ILO (International Labour Office), 1980. Guideline for Use of ILO International Classification of Radiographs of Pneumoconiosis. Occupational Safety and Health Series 22. Geneva: World Health Organization.

ISAZA, D.; RAMIREZ, R.; FRANCO, S.; DURAN, C. J. \& TAPIAS, B., 1988. Morbilidad respiratoria en trabajadores de la industria del talco. Iatreia, 1:2228.

JONES, N. R.; WEILL, H. \& PARKES, W. R., 1994. Disease related to non-asbestos silicates. In: Occupational Lung Disorders (R. Parkes, ed.), pp. 536550, 3rd Ed., Oxford: Butterworth Heinemann.

KNUDSON, R. J.; SLATIN, R. C.; LEIBOWITZ, M. D. \& BURROWS, B., 1976. The maximal expiratory flow-volume curve: Normal standards, variability and effects of age. American Review of Respiratory Disease, 113:587-600.

MENEZES, A. M. B.; VICTORA, C. G. \& RIGATTO, M., 1994. Prevalence and risk factors for chronic bronchitis in Pelotas, RS, Brazil: A populationbased study. Thorax, 49:1217-1221.

MOREIRA, L. B. \& FUCHS, F. D., 1995. Prevalência de tabagismo e fatores associados em área metropolitana da Região Sul do Brasil. Revista de Saúde Pública, 29:46-51.

MORGAN, W. K. C. \& SEATON, A., 1995. Occupational Lung Diseases. Philadelphia: WB Saunders.

PARKES, W. R., 1973. Asbestos-related disorders. British Journal of Diseases of Chest, 67:261-300.

PEREIRA, C. A. C.; BARRETO, S. P.; SIMÕES, J. G.; PEREIRA, F. W. L.; GERSTLER, J. G. \& NAKATANI, J., 1992. Valores de referência para a espirometria em uma amostra da população brasileira adulta. Jornal de Pneumologia, 18:10-22.

PIVETTA, A. B. D. A. \& BOTELHO, C., 1997. Prevalência de sintomas respiratórios e avaliação espirométrica em trabalhadores de marmoarias. Jornal de Pneumologia, 23:179-188.

POLGAR, P. \& PROMADHAT, P., 1971. Pulmonary Testing in Children. Philadelphia: WB Saunders.

RAM OS, M. C., 1983. Sintomas respiratórios na população da cidade de Ribeirão Preto, SP (Brasil). Resultados da aplicação de um questionário padronizado. Revista de Saúde Pública, 17:41-50.

SOCIEDADE BRASILEIRA DE PNEUMOLOGIA E TISIOLOGIA, 1996. I Consenso Brasileiro sobre Espirometria. Jornal de Pneumologia, 22:105-164.

WEGM AN, D. H.; PETERS, J. M.; BOUNDY, M. G. \& SMITH, T. J., 1982. Evaluation of respiratory effects in miners and millers exposed to talc free of asbestos and silica. British Journal of Industrial Medicine, 39:233-238.

Recebido em 12 de setembro de 2002

Versão final reapresentada em 22 de maio de 2003

Aprovado em 4 de agosto de 2003 